\title{
A curved-grid gas proportional scintillation counter instrumented with a 25-mm active-diameter photosensor
}

\author{
C.M.B. Monteiro ${ }^{\mathrm{a}}$, R.E. Morgado ${ }^{\mathrm{b}}$, J.M.F. dos $\operatorname{Santos}^{\mathrm{a}, *}$, C.A.N. Conde ${ }^{\mathrm{a}}$ \\ ${ }^{a}$ Physics Department, University of Coimbra, Coimbra P-3004-516, Portugal \\ ${ }^{\mathrm{b}}$ Los Alamos National Laboratory, Los Alamos, NM, USA
}

Received 2 July 2003; received in revised form 23 October 2003; accepted 29 November 2003

\begin{abstract}
The curved-grid technique provides a simple method to compensate for the variation in solid angle viewed by the PMT in large-area gas proportional scintillation counters (GPSC), improving their performance. The scintillation region is delimited by a planar grid and a curved one, whose shape is calculated to produce a radially increasing scintillation yield, compensating for the decrease in the solid angle. In this work, we applied this technique to a GPSC instrumented with a PMT having a $25-\mathrm{mm}$ diameter photocathode. The maximum ratio of the detector entrance window to the photocathode diameter thus far achieved, without significant performance degradation, is 1 .
\end{abstract}

(C) 2004 Elsevier B.V. All rights reserved.

PACS: $07.85 . \mathrm{Nc} ; 29.40 . \mathrm{Cs} ; 29.40 .-\mathrm{n}$

Keywords: Gas scintillation counter; Xenon gas detector; X-ray detector

\section{Introduction}

Amongst all X-ray gas detectors, gas proportional scintillation counters (GPSCs) provide the best performance [1]. Compared to proportional counters, they deliver superior energy resolution and higher count-rate capability without spacecharge effects. These features result from the characteristic amplification of the primary ionisation charge by means of scintillation processes in noble gases, with reduced statistical fluctuations and no space-charge build-up, in contrast to the charge avalanche process used in other type of gas

\footnotetext{
*Corresponding author. Tel.: + 1-351-2934-106-67; fax: + 1351-239-829-158.

E-mail address: jmf@gian.fis.uc.pt (J.M.F. dos Santos).
}

detectors. Additionally, the achieved large scintillation gain, combined with a photosensor with suitable sensitivity (e.g. photomultipliers), allows to obtain detector pulses with large amplitudes, more than one order of magnitude larger than those obtained with proportional counters.

The theory and operation of the GPSC are reviewed in a recent publication [2]. A typical GPSC consists of a gas volume divided into two regions: the absorption and the scintillation regions. A photosensor, usually a photomultiplier tube (PMT), records the scintillation light produced in the scintillation region (Fig. 1). X-ray photons absorbed in the drift region by the photoelectric effect produce a primary electron cloud that drifts towards the scintillation region under the influence of a weak electric field chosen 


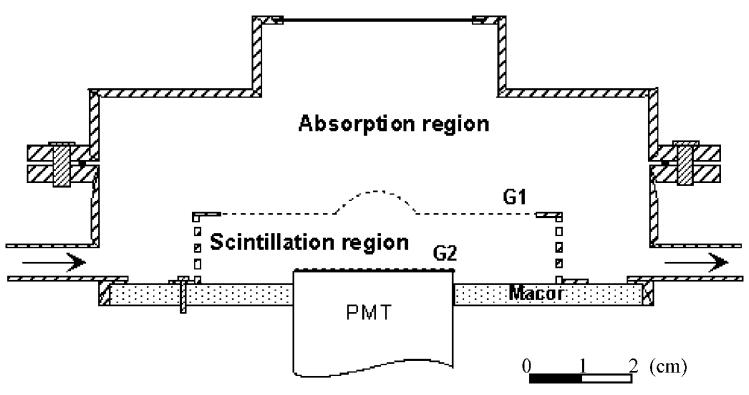

Fig. 1. Schematic diagram of the curved-grid GPSC used in the present work.

well below the gas scintillation threshold. In the scintillation region, however, the electric field is chosen to be above the gas scintillation threshold, but below the ionisation threshold. In the scintillation region, the electrons' kinetic energies are high enough to excite but not ionise the gas atoms, giving rise to a large number of VUV photons, as a result of the atoms' de-excitation processes, without undergoing charge avalanche amplification. The total number of VUV photons is proportional to the number of primary electrons entering the scintillation region and, thus, to the energy of the incident X-ray, independent of where the X-ray interaction occurred. In contrast to charge avalanche processes, the statistical fluctuations associated with the light-amplification process are negligible compared to those associated with the formation of the primary electron cloud. Thus, the GPSC energy resolution approaches its intrinsic value, provided that the fluctuations associated with the photosensor are also kept low.

As the GPSC-sensitive area is increased, energy resolution deteriorates with the larger solid angle over which the scintillation light must be collected. Pulse amplitude will depend on the radial distance from the detector axis, $r$, where the electroluminescence takes place and, thus, on the X-ray interaction position, since the solid angle viewed by the photosensor, will also vary as function of $r$. This is the most limiting effect on the energy resolution for GPSCs with medium and large detection areas compared to the area of the photosensor.

Methods to minimize this effect were developed [3-6]. Focusing electrodes and/or spherical electric fields in the drift region have been used to drive the primary electrons to the region around the detector axis prior to entering the scintillation region. Additionally, in the spherical-field design, the scintillation-region-to-photosensor distance decreases radially, partially compensating for the decrease in solid angle with increasing radius. The spherical-field design allows the use of detection areas that are significantly larger than the photosensor area $[4,5]$.

More recently, a simpler method, the curvedgrid technique [7,8], was developed to compensate for the variation in solid angle viewed by the PMT, as a function of radial distance from the cylindrical axis of the detector. In large-area GPSCs, the curved-grid technique eliminates the need for complex and bulky focusing systems or for large PMTs. A curved grid and a planar grid are used to delimit the scintillation region, instead of the two parallel grids usually used in GPSCs. The shape of the curved grid is calculated to produce a radially increasing electric field in the scintillation region (Fig. 1), which results in a radial increase of the scintillation yield, since this yield depends on the electric field [2]. A correctly shaped curved grid will produce an increasing scintillation yield with increasing radius that compensates for the reduction in the solid angle viewed by the photosensor. The technique was shown to successfully compensate solid angle effects for detector window diameters up to $80 \%$ of the photocathode diameter, in GPSCs instrumented with PMTs having 48- $\mathrm{mm}$ [9] and 33-mm [8] active diameters. The experimental results obtained in Ref. [8] suggested that good solid angle compensation could be achieved for even larger radial distances.

In the present work, we apply the curved-grid technique to a GPSC instrumented with a PMT having a $25-\mathrm{mm}$ active diameter photocathode. In contrast to the previous studies [7-9], in the present work, we used a detector window with a larger diameter than that of the PMT in order to evaluate the full capability of this technique.

\section{Method description}

Assuming an electron path parallel to the detector axis in the scintillation region, the average 
solid angle, $\Omega_{\mathrm{r}}$, subtended by the photosensor as function of the radial distance can be determined by either Monte Carlo simulation or numerical calculation [10]. In order to compensate for the variation in solid angle, the number of scintillation photons reaching the photosensor should be independent of the radial position of the electron path. Thus, we need to have

$N_{\mathrm{r}} \Omega_{\mathrm{r}}=N_{0} \Omega_{0}$

where $N_{0}$ and $N_{\mathrm{r}}$ are the number of photons produced by an electron along the axis and along a radial distance $r$, and $\Omega_{0}$ and $\Omega_{\mathrm{r}}$ are the respective average solid angles subtended by the photosensor for each path.

Since the reduced electroluminescence yield (the yield divided by the gas pressure, $p$ ) has a linear dependence on the reduced electric field (the electric field divided by $p$ ) [2], with a threshold at $1 \mathrm{~V} \mathrm{~cm}^{-1} \mathrm{Torr}^{-1}$, we can write

$\frac{N_{\mathrm{r}}}{p d_{\mathrm{r}}}=a \frac{E}{p}+b$

$\frac{N_{\mathrm{r}}}{p d_{\mathrm{r}}}=a \frac{V}{p d_{\mathrm{r}}}+b \Rightarrow N_{\mathrm{r}}=a V+b p d_{\mathrm{r}}$

assuming a constant electric field, $V / d_{\mathrm{r}}$, along the electron path, to be a good approximation. Under these conditions, the inter-grid distance $d_{\mathrm{r}}$ can be written as

$$
\begin{aligned}
d_{\mathrm{r}} & =\frac{N_{\mathrm{r}}-a V}{b p}=\frac{N_{0} f_{\mathrm{r}}-a V}{b p}=\frac{\left(a V+b p d_{0}\right) f_{\mathrm{r}}-a V}{b p} \\
& =d_{0} f_{\mathrm{r}}+\left(f_{\mathrm{r}}-1\right) \frac{V}{p} \frac{a}{b}
\end{aligned}
$$

where $f_{\mathrm{r}}=\Omega_{0} / \Omega_{\mathrm{r}}$. As $N=0$ at the scintillation threshold, $(E / p)_{0} \approx 1 \mathrm{~V} \mathrm{~cm}^{-1}$ Torr $^{-1}$; from Eq. (2) we obtain $a / b=-(E / p)_{0}^{-1} \approx-1 \mathrm{~V} \mathrm{~cm}^{-1} \mathrm{Torr}^{-1}$.

The relative solid angle variation, $\Omega_{\mathrm{r}} / \Omega_{0}$, is obtained using Monte Carlo simulation described in Ref. [10]. In Fig. 2a, $\Omega_{\mathrm{r}} / \Omega_{0}$ is presented as function of $r$, for a $13-\mathrm{mm}$ thick scintillation region and a $25-\mathrm{mm}$ diameter photocathode. In Fig. 2b, the inter-grid distance, obtained using the data of Fig. 2a in Eq. (3), is depicted as function of $r$, for a voltage difference of $5 \mathrm{kV}$ and for a $\mathrm{Xe}$ pressure of 1120 mbar. The solid lines present 8-, 9- and 10-mm-radius spherical shapes. Although the best approximation would be to the radii of 8
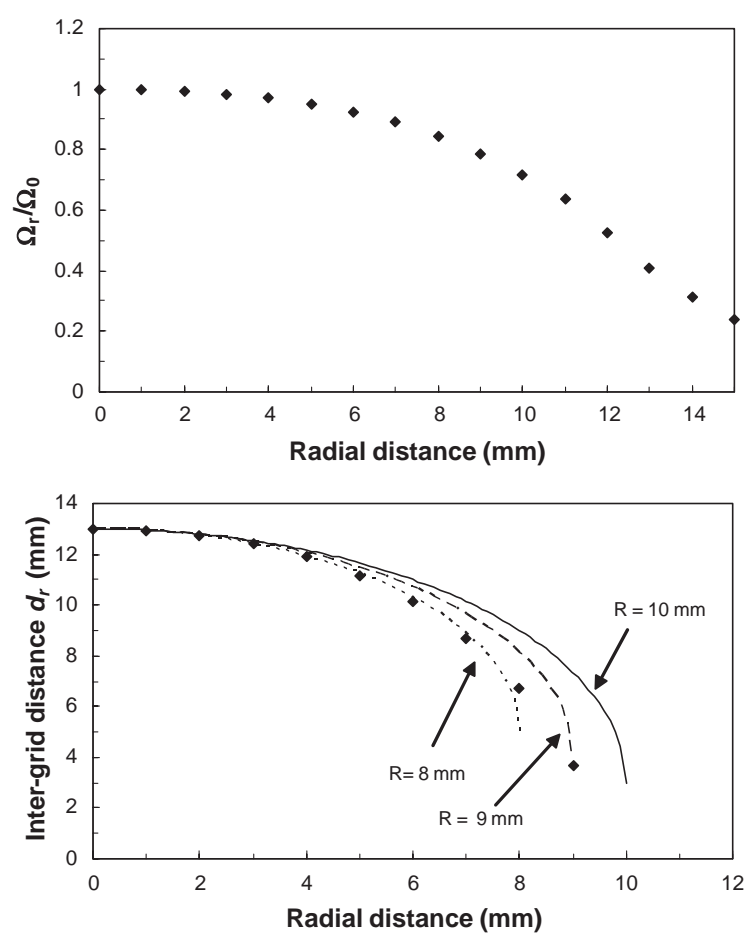

Fig. 2. (a) Relative variation of the solid angle subtended by a $25-\mathrm{mm}$ photosensor as function of the radial distance, $r$, for a 13-mm long trajectory above the photosensor parallel to the photosensor axis. (b) Calculated inter-grid spacing as function of $r$ for solid angle effect compensation; the depicted solid lines are arcs of circles of 8-, 9- and 10-mm radii.

$9 \mathrm{~mm}$, a radius of $10 \mathrm{~mm}$ was chosen for easier grid-mould manufacturing and also to reach solidangle compensation for higher radial distances.

The method to calculate the shape of the curved grid presented in this work differs from that described in Ref. [8], and requires less computing, though both methods lead to similar results.

\section{Experimental set-up}

The detector is depicted schematically in Fig. 1. We have chosen $d_{0}=13 \mathrm{~mm}$, and a $10-\mathrm{mm}$-radius spherical shape was found to be a reasonable approximation to the calculated $d_{\mathrm{r}}$ values. Thus, the depth of the scintillation region varies from $13 \mathrm{~mm}$ at the detector axis to $6 \mathrm{~mm}$ in the planar lateral region and the grid curvature extends to radial distances of $9.5 \mathrm{~mm}$. The drift region 
thickness varies from $30 \mathrm{~mm}$ at the detector axis to $37 \mathrm{~mm}$ in the planar lateral region.

The detector body is a stainless-steel cylinder, while the bottom part is a Macor disc used to insulate grids G1 and G2 (the low- and highvoltage grids, respectively). The Macor is glued with epoxy to the detector wall and to the PMT. The stainless-steel support of G1 is fixed to the Macor with screws, one of which is used as a feedthrough for the G1-bias voltage. The upper and lower parts of the detector are sealed by compression of an indium gasket. The detector radiation window is a $50-\mu \mathrm{m}$ Kapton film, aluminised on the inner surface, and glued with Epoxy to the detector body. The PMT (EMI 9125QB) has a diameter of $30 \mathrm{~mm}$, an 11-dynode linear-focused structure and a 25-mm-diameter photocathode. The detector was filled with pure xenon at 1120 mbar, continuously purified by convection through non-evaporable getters at $150-200^{\circ} \mathrm{C}$.

The curved grid G1 is a stainless-steel mesh with $80-\mu \mathrm{m}$-diameter wire and $900-\mu \mathrm{m}$ spacing. Its shape is obtained by compression in a mould. The planar grid G2 was obtained by vacuum thermal deposition of chromium on the PMT window, and consists of $100-\mu \mathrm{m}$-wide lines at 1 $\mathrm{mm}$ spacing. A chromium film was also evaporated on the lateral surface of the PMT in order to obtain a homogeneous electric field in the scintillation region and to establish an electric connection between G2 and the PMT photocathode pin. In this way, there is no potential difference between the photocathode and the external surface of the PMT window. Thus, the PMT is polarized at high voltage, floating between $\mathrm{G} 2$ high voltage and the anode high voltage, $V_{\mathrm{G} 2}+V_{\mathrm{PMT}}$.

For the present work, 5.9-keV X-rays emitted by a ${ }^{55} \mathrm{Fe}$ radioactive source were used. The $6.4-\mathrm{keV}$ $\mathrm{Mn} \mathrm{K}_{\beta}$-line was filtered through a chromium film. The detector pulses were fed through a preamplifier and a linear amplifier to a 1024 multi-channel analyser. To determine the peak amplitude and the energy resolution, pulse-height distributions were fitted to a Gaussian superimposed on a linear background from which the centroid and the full-width-at-half-maximum were determined.

\section{Experimental results and discussion}

Fig. 3 depicts the relative pulse-height amplitude and energy resolution as functions of the G1 voltage, while maintaining grid $\mathrm{G} 2$ at $5.0 \mathrm{kV}$. The best detector performance is achieved for G1 voltages around $250 \mathrm{~V}$, corresponding to a reduced electric field of about $0.1 \mathrm{~V} \mathrm{~cm}^{-1} \mathrm{Torr}^{-1}$, in the drift region.

To evaluate the capability of this design to compensate for solid angle variations, we investigated the detector relative pulse-height amplitudes, Fig. 4, and energy resolution, Fig. 5, as function of the detector window diameter, for G2 voltages of 5.0 and $5.3 \mathrm{kV}$, while maintaining G1 at $250 \mathrm{~V}$. Lead collimators covering the detector radiation window determined the different radiation window diameters. The distance of the radioactive source to the detector window was increased with the window diameter, in order to maintain similar count rates for all cases.

As pointed out in Ref. [7], the compensation condition calculated through Eq. (4) is valid only for a certain voltage difference, $V_{\mathrm{G} 2}-V_{\mathrm{G} 1}$, given a certain geometry and gas pressure. Good compensation is achieved for $\mathrm{G} 2$ voltages around $5.3 \mathrm{kV}$, as evidenced by the independence of the pulseheight amplitude and energy resolution of the detector window diameter. At this voltage, the pulse amplitudes are independent of the detector window diameter, up to window diameters of $25 \mathrm{~mm}$, decreasing above this value. On the other

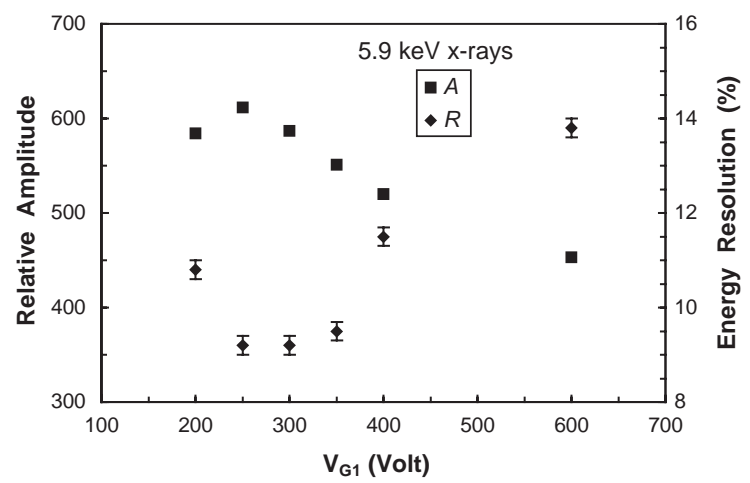

Fig. 3. Detector relative pulse amplitude, $A$, and energy resolution, $R$, as function of grid G1 voltage, for a constant $\mathrm{G} 2$-voltage of $5.0 \mathrm{kV}$. 


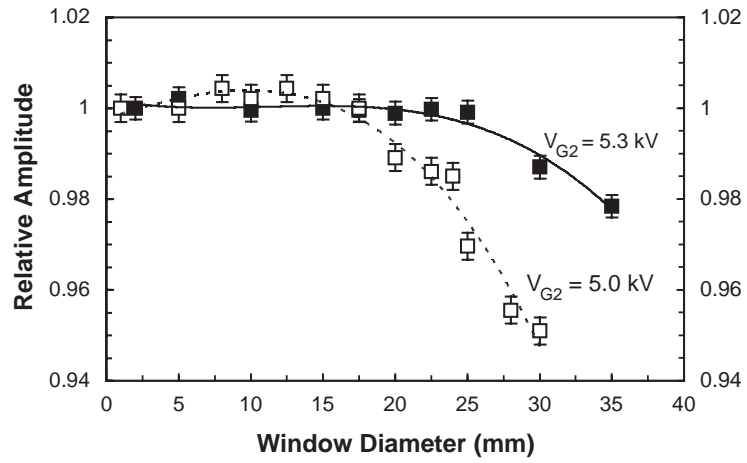

Fig. 4. Detector relative pulse amplitude as a function of radiation window diameter for grid G2 voltages of 5.0 and $5.3 \mathrm{kV}$. The lines serve only to guide the eye.

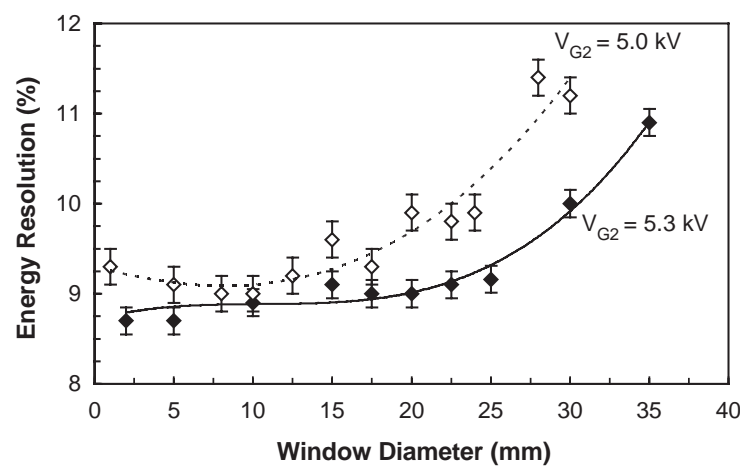

Fig. 5. Detector energy resolution as a function of radiation window diameter for 5.9-keV X-rays and for G2 voltages of 5.0and $5.3-\mathrm{kV}$. The lines serve only to guide the eye.

hand, energy resolution increases slowly from $8.7 \%$ to $9.1 \%$ as the window diameter increases from 2 to $25 \mathrm{~mm}$, degrading rapidly above this value. For $\mathrm{G} 2$ voltages above $5.3 \mathrm{kV}$, the detector performance degrades due to the onset of electric discharges.

The curved-grid technique allows building detectors with detection areas equal to the photosensor active area, without significant degradation of its energy resolution. This represents a significant improvement over the GSPC described in Ref. [11], which has parallel grids, only one focusing electrode, and a PMT with a 33-mm diameter photocathode. The energy resolution obtained with this detector was $8.3 \%$ for $5.9-\mathrm{keV}$ $\mathrm{X}$-rays, degrading to $8.6 \%, 8.8 \%$ and $9.2 \%$ for radiation window diameters of only 10,16 and $20 \mathrm{~mm}$ (i.e., detection areas of $9 \%, 24 \%$ and $37 \%$ of the photosensor active area), respectively, and increasing rapidly above $20 \mathrm{~mm}$. With the present detector, an energy resolution of $9.1 \%$ was obtained for a radiation window diameter of $25 \mathrm{~mm}$. Additionally, with the curved-grid GPSC of Ref. [8], which has a 33-mm active-diameter photosensor, an energy resolution of $8.5 \%$ was obtained for a radiation window diameter of $25 \mathrm{~mm}$ and, from its performance, one can expect an energy resolution below $9 \%$ for radiation window diameters around $33 \mathrm{~mm}$ (i.e., a detection area of $100 \%$ of the photosensor active area).

In Fig. 6, we depict the equipotential curves of the electric fields inside the detector, as obtained by a two-dimensional electric field simulator [12], considering the detector body at $0 \mathrm{~V}$, the curved grid at $300 \mathrm{~V}$ and the PMT enclosure at $5300 \mathrm{~V}$. In the region near the grid at the detector axis, the reduced electric field inside the grid curvature reaches values as low as $2.5 \mathrm{~V} \mathrm{~cm}^{-1} \mathrm{Torr}^{-1}$. As shown, the small radius of the curved grid results in an electric field with reduced intensity inside the curvature. This effect may be responsible for the low voltage requirement of G1 as well as for the higher energy resolution achieved with this detector and that of Ref. [8], which uses a 12-mm radius spherical grid, compared to the values achieved with a $20-\mathrm{mm}$ grid curvature (e.g. $8.0 \%$ for 5.9-keV X-rays) [7]. The use of elliptically

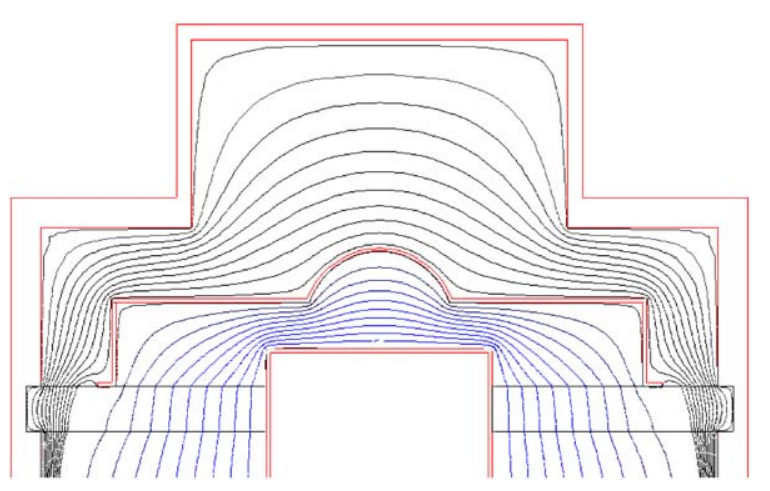

Fig. 6. Schematic presentation of the equipotential field lines for the electric fields inside the detector and the Macor ceramic. While for the drift region the equipotential lines are presented in $\sim 29-\mathrm{V}$ gap, for the scintillation region the gap is $\sim 500 \mathrm{~V}$. 


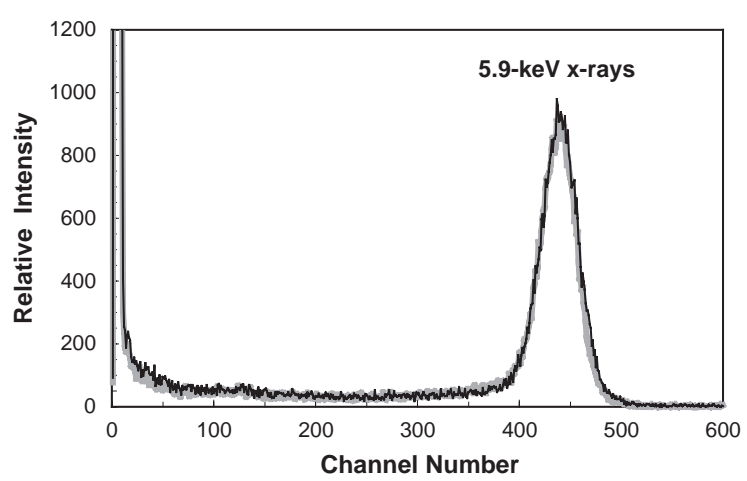

Fig. 7. Pulse-height distributions obtained with the curved-grid GPSC for 5.9-keV X-rays and for detector radiation window diameters of 2- and $25-\mathrm{mm}$, thick grey line and thin black line, respectively.

shaped grids $[9,13]$ that fit the calculated $d_{\mathrm{r}}$ values to a reasonable approximation can improve the performance of this technique, since the smaller grid curvature leads to more intense electric fields inside the curvature.

In Fig. 7 we depict the pulse-height distributions obtained for $5.9-\mathrm{keV}$ X-rays and for detector radiation window diameters of 2 - and $25-\mathrm{mm}$, grey and black lines, respectively. No degradation is apparent, either in energy resolution, background level, or electronic noise tail at the lowenergy limit. For the latter case, the ratio of detector entrance window- to-photosensor-activediameter is 1 .

\section{Conclusions}

We have demonstrated that the curved-grid technique compensates solid angle effects for detector radiation window diameters up to values similar to those of the photosensor active diameter. Above these values, the compensation is no longer effective and the detector performance degrades rapidly. With this technique, it is possible to produce simple, high-performance GPSCs with radiation window areas similar to those of the photosensor, without resorting to complex and bulky focusing systems. A new method to calculate the shape of the curved grid was presented.
A spherical curved grid with a $10-\mathrm{mm}$ radius is suitable to compensate the solid angle effects of 25-mm diameter photosensors. This could be important in applications where large-area avalanche photodiodes are considered to substitute for PMTs. Further improvements can be obtained using ellipsoidal shapes for the curved grid.

\section{Acknowledgements}

This work has been carried out in the Instrumentation Centre (unit 217/94) of the Physics Department, University of Coimbra. Support is acknowledged to Fundação para a Ciência e a Tecnologia (FCT) through project POCTI/FIS/ 43527/99. Thanks are due to D.S.A.P. Freitas (GIAN, University of Coimbra) for his help with the electric field calculations.

\section{References}

[1] G.F. Knoll, Radiation Detection and Measurements, 3rd Edition, Wiley, New York, 2000.

[2] J.M.F. dos Santos, J.A.M. Lopes, J.F.C.A. Veloso, et al., X-ray Spectrom. 30 (2001) 373.

[3] D.F. Anderson, O.H. Bodine, R. Novick, R.S. Wolff, Nucl. Instr. and Meth. A 144 (1977) 485.

[4] D.F. Anderson, W.H.M. Ku, R. Novick, M. Scheckman, IEEE Trans. Nucl. Sci. NS-25 (1978) 813.

[5] R.D. Andresen, E.A. Leimann, A. Peacock, B.G. Taylor, IEEE Trans. Nucl. Sci. NS-25 (1978) 800.

[6] D.A. Goganov, A.A. Shultz, V.B. Elkind, Instr. Exp. Techn. (USSR) 27 (1984) 478.

[7] C.A.N. Conde, J.M.F. dos Santos, A.C.S.S.M. Bento, IEEE Trans. Nucl. Sci. NS-40 (1993) 452.

[8] J.M.F. dos Santos, A.J.V.D. Soares, C.M.B. Monteiro, R.E. Morgado, C.A.N. Conde, IEEE Trans. Nucl. Sci. NS-45 (1998) 229.

[9] R.M.C. Silva, J.M.F. dos Santos, C.A.N. Conde, Nucl. Instr. and Meth. A 422 (1999) 305.

[10] J.M.F. dos Santos, A.C.S.S.M. Bento, C.A.N. Conde, IEEE Trans. Nucl. Sci. NS-39 (1992) 541.

[11] D.A. Goganov, A.A. Shultz, Nucl. Instr. and Meth. A 394 (1997) 151

[12] Maxwell 2D Field Simulator-Ansoft Coorporation, Four Station Square, Suite 660, Pittsburgh, PA 151219 (Tel.: + 1-412-261-3200).

[13] H. Natal da Luz, J.F.C.A. Veloso, J.M.F. dos Santos, C.A.N. Conde, et al., IEEE Trans. Nucl. Sci. NS-49 (2002) 2488. 